\title{
Tilt control of the charged domain walls in lithium niobate
}

\author{
A.R. Akhmatkhanov, A.A. Esin, V.Ya. Shur
}

School of Natural Sciences and Mathematics, Ural Federal University, 620000, Ekaterinburg, Russia Andrey.Akhmatkhanov@urfu.ru

Precise control of the domain structure in ferroelectric single crystals is one of the most important and challenging tasks in physics of ferroelectrics. So far, main part of investigations in this area was aimed at realization of high efficiency nonlinear optical interactions, such as second harmonic generation (SHG) and optical parametric oscillation. These applications require precise spatial variation of the spontaneous polarization, which distribution within the crystal is determined by the positions and orientations of the domain walls (DWs). Recently, the scientific attention has turned from the domains to the DWs themselves: these movable unit-cell-thick interfaces were proposed as the building blocks for reconfigurable nanoelectronics devices, because their properties can differ drastically from the bulk parent material [1,2]. In particular, it has been shown that conductivity of the charged domain walls (CDWs) in ferroelectrics is many orders of magnitude higher, than that in the single domain state [3]. The utilization of the CDW as nanoelectronics devices requires three technological aspects to be developed: (1) controllable creation, (2) geometry tuning, and (3) removal.

We present the experimental study of the CDW formation in congruent lithium niobate single crystals during polarization reversal using liquid and solid-state electrodes, as well as their combination [4]. It was shown that material of the electrode applied to the Z-polar surface is crucial. It was shown that $\mathrm{CDW}$ can be formed by two alternative procedures: (1) by forward switching for liquid electrode at Z+ polar surface and solid electrode - at Z- one, (2) by backward switching for solid electrode at Z+ polar surface and liquid electrode at Z- one. The obtained domain structure was investigated in the bulk of the crystal using scanning Cherenkov-type SHG microscopy [5]. For both used procedures the CDWs were formed when domains grew from Z+ polar surface covered by solid or liquid electrode towards Z- surface covered by solid electrode. Thus, existence of the solid electrode on Z- surface is the necessary condition for CDW formation. The created CDW can be transformed to almost neutral domain wall by application of the field pulse of the reverse polarity for both procedures. As a result, the tilt of the formed CDW can be tuned reversibly in the range from 0.2 to 1.2 degrees resulting in change between almost isolated and highly conductive states. When the tilt exceeded 1.2 degrees the CDW became jugged due to formation of additional spikes. For the second procedure the unusual partial backward switching during forward polarization reversal was revealed. Moreover, we have demonstrated that the created CDW can be used as a nanoelectronics channel for local electrolysis opening the additional possibilities for the ferroelectric lithography.

The equipment of the Ural Center for Shared Use "Modern nanotechnology" Ural Federal University was used. The research was made possible in part by RFBR (grant 18-3200641_mol_a).

1. A. Crassous, T. Sluka, A.K. Tagantsev, N. Setter, Nat. Nanotechnol. 10, 614 (2015).

2. P. Sharma, Q. Zhang, D. Sando, C.H. Lei, Y. Liu, J. Li, V. Nagarajan, J. Seidel, Sci. Adv. 3, e1700512 (2017).

3. V.Ya. Shur, I.S. Baturin, A.R. Akhmatkhanov, D.S. Chezganov, A.A. Esin, Appl. Phys. Lett. 103, 102905 (2013).

4. A.A. Esin, A.R. Akhmatkhanov, V.Ya. Shur, Appl. Phys. Lett. 9, 092901 (2019).

5. Y. Sheng and A. Best, Optics Express 18, 16 (2010). 